\title{
Descendit de Caelis. Liturgia, arquitectura y teatro en los monasterios de las dominicas castellanas en la Baja Edad Media
}

\author{
Mercedes PÉREZ VIDAL \\ Universidad Nacional Autónoma de México \\ mercedesvidal24@gmail.com
}

\section{RESUMEN}

Tanto la liturgia, como las paraliturgias o representaciones asociadas a ella, desarrolladas en los monasterios femeninos de la Península Ibérica, resultan todavía aspectos bastante desconocidos. Sin embargo, a través de una metodología integradora y novedosa en el estudio del monacato femenino hispano, se pueden reconstruir algunas de estas prácticas, evidenciando el papel de las monjas en su definición y celebración, la participación de diversos objetos y los espacios en los que tenían lugar estas representaciones. Asimismo, en contra de la consideración tradicional de la preeminencia franciscana en los orígenes del teatro religioso, se evidencia el papel que Dominicas y Dominicos tuvieron en su desarrollo.

Palabras clave: Dominicas; Castilla; liturgia y arquitectura; teatro; Reforma; Baja Edad Media.

\section{“Descendit de Caelis". Liturgy, Architecture and Theatre in the Domini- can Castilian Nunneries in the Late Middle Ages}

\begin{abstract}
Both the liturgy and paraliturgies or ceremonies linked to it within the nunneries in the Iberian Peninsula still remain almost unknown. However, by applying a new and integrating approach, which has made little impact in the historiography of women's monasticism in this area, we can trace the development of some of these practices, as well as the role of nuns in their shaping and performing, the use of some artifacts, and the spaces in which representations were carried out. Additionally, opposed to the traditional consideration of the Franciscan preeminence in the origins of religious plays, I argue the role of Dominican nuns and friars in their development.
\end{abstract}

Key words: Dominican nuns; Castile; Liturgy and architecture; Theatre; Reform; Late Middle Ages. 
La descentralización de la historia propia de la postmodernidad y la renovación y ampliación del horizonte metodológico que supusieron la Cultura Visual y los Estudios Visuales, ha acarreado no solo la floración de estudios inter o multidisciplinares, sino también un creciente interés por áreas hasta entonces descuidadas. En concreto, y centrándonos en lo que aquí nos interesa, a partir de la década de los ochenta del siglo pasado, y más aún en la siguiente, tuvo lugar un notable despegue de la historiografía sobre el monacato femenino, que se insertó a su vez dentro de un enfoque feminista más amplio que, si bien con altibajos, sigue aún vigente ${ }^{1}$. Este interés por las monjas medievales no se ha dado por igual en todos los ámbitos geográficos y el acercamiento metodológico ha sido sumamente variado, desde un enfoque próximo a la historia social-centrado en la relación de los monasterios con el entorno- a la reconstrucción del ambiente cultural, la espiritualidad, devociones y la cultura visual de las religiosas ${ }^{2}$. Lógicamente, esto último ha sido posible gracias a la recuperación de las voces, hasta entonces silenciadas, de las religiosas medievales, a través de la edición y estudio de sus escritos hagiográficos y devocionales ${ }^{3}$.

1 BLEEKE, Marian, "Feminist Approaches to Medieval Visual Culture: An Introduction", en Medieval Feminist Forum, no 44.2, 2008, pp. 49-52.

2 Resulta imposible incluir en este limitado espacio una relación exhaustiva de estas publicaciones. No obstante, algunos ejemplos relevantes para lo que se tratará aquí son: WOOD, Jeryldene M., Women, Art, and Spirituality. The Poor Clares of Early Modern Italy, Cambridge, Cambridge University Press, 1996; HAMBURGER, Jeffrey F., Nuns as Artists: The Visual Culture of a Medieval Convent, Berkeley, University of California Press, 1997; Idem, The Visual and the Visionary: Art and Female Spirituality in Late Medieval Germany, Cambridge, MA, MIT Press, 1998; LEE, Paul, Nunneries, Learning and Spirituality in late medieval English society. The dominican priory of Dartford, York Medieval Press, 2001; BLONDEL, Madeleine; HAMBURGER, Jeffrey. F. y LEROY, Catherine (eds.), Les dominicaines d Unterlinden (Musée de Unterlinden, Colmar, 2000-2001), Paris, Somogy, éditions d’art, 2000; THOMAS, Anabel, Art and Piety in the Female Religious Communities of Renaissance Italy. Iconography, Space, and the Religious Women's Perspective, Cambridge, Cambridge University Press, 2003; HAMBURGER, Jeffrey. F. y MARTI, Susan (eds.), Krone und Schleier: Kunst aus mittelalterlichen Frauenklöstern, München, Hirmer Verlag, 2005. reed. en inglés como Crown and Veil, Female Monasticism from the Fifth to the Fifteenth Centuries, New York, Columbia University Press, 2008; HAMBURGER, Jeffrey F., JÄGGI, Carola, MARTI, Susan y RÖCKELEIN, Hedwig (eds.), Frauen - Kloster - Kunst: Neue Forschungen zur Kulturgeschichte des Mittelalters. Internationales Kolloquium im Zusammenhang mit Krone und Schleier: Kunst aus mittelalterlichen Frauenklöstern, Die Wolfsburg, Mülheim/Ruhr, Turnhout, Brepols, 2007.

3 DRONKE, Peter, Women Writers of the Middle Ages: a Critical Study of Texts from Perpetua (d. 203) to Marguerite Porete (d. 1310), Cambridge, Cambridge University Press, 1984; SURTZ, Ronald E., Writing women in late Medieval and early modern Spain: the mothers of Saint Teresa of Avila, University of Pennsylvania Press, 1995; WILKINS, Constance. L., "El devocionario de Sor Constanza: otra voz femenina medieval", en Actas del XII Congreso de la Asociación Internacional de Hispanistas, Birmingham, 1998, t. I, pp. 340-349; RUCQUOI, Adeline, "Lieux de spiritualité féminine en Castille au XVe siècle", en Via Spiritus, vol. 7, 2000, pp. 7-29; WINSTON-ALLEN, Anne, Convent Chronicles. Women Writing About Women and Reform in the Late Middle Ages, The Pennsylvania State University Press, 2004; CLASSEN, Albert, The Power of a Woman's Voice in Medieval and Early Modern Literatures, Berlín, The Gruyter, 2007; MINNIS, Alastair y VOADEN, Rosalynn (eds.), Medieval Holy Women in the Christian Tradition, c. 1100-c. 1500, Turnhout, Brepols, 2010; ZARRI, Gabriella y BARANDA LETURIO, Nieves (coords.), Memoria e Comunità Femminili. Spagna e Italia, sec. XV-XVII. Memoria y comunidades femeninas. España e Italia, siglos XV-XVII, Firenze University Press, UNED, 2011; MOITEIRO, Gilberto Coralejo, As dominicanas de Aveiro (c. 14501525): Memória e identidade de uma comunidade textual, Tese de Doutoramente em Historia, Universidade Nova de Lisboa, 2013, inédita. Agradezco a Gilberto Moiteiro su gentileza al facilitarme su tesis. 
Por el contrario, y pese a constituir un elemento clave en la vida religiosa, la liturgia propia de las monjas continúa siendo bastante desconocida. A finales de los noventa Teresa Berger señalaba como hasta entonces apenas un puñado de publicaciones se habían ocupado de la vida litúrgica de las mujeres, mientras el resto continuaba considerando el género una cuestión irrelevante ${ }^{4}$. La respuesta a esta llamada de atención ha sido, sin embargo, lenta y desigual según los ámbitos geográficos. En relación a los asuntos aquí tratados, cabe destacar especialmente los trabajos de Gisela Muschiol y Carola Jäggi, que se insertan además en el llamado "giro litúrgico", esto es el estudio del arte y la arquitectura en relación a la liturgia ${ }^{5}$.

Pero además, cabe tener presente que las interpolaciones en el oficio divino, y las procesiones y paraliturgias asociadas a las principales festividades estuvieron en el origen no solo del drama litúrgico, sino también del teatro vernáculo. Pedro Cátedra ha puesto de manifiesto recientemente el papel que tuvieron las monjas castellanas-y en concreto, las comunidades de clarisas- en el desarrollo de esta tradición teatral medieval, que fue renovada y actualizada en los albores de la Edad Moderna, momento en el que tales prácticas aparecen ya sobradamente documentadas en las clausuras ${ }^{6}$.

Lamentablemente, en el caso de las fundaciones femeninas de la Península Ibérica, tanto estas prácticas, como la liturgia creada por y para las mujeres resultan todavía aspectos bastante desconocidos $y$, en consecuencia, el estudio conjunto del arte, arquitectura y liturgia apenas ha tenido incidencia en este ámbito ${ }^{7}$.

4 BERGER, Teresa, Women 's Ways of Worship: Gender Analysis and Liturgical History, Collegeville, Minn. Liturgical Press, 1999.

5 MUSCHIOL, Gisela, "Liturgie und Klausur. Zu den liturgischen Voraussetzungen von Nonnenchören”, en CRUSIUS, Irene (ed.), Studien zum Kanonissenstifte, Göttigen, Studien zu Germani Sacra- Veröffentlichungen des Max-Planck-Instituts für Geschichte, 2001, pp. 129-148; MUSCHIOL, Gisela, "Time and Space: Liturgy and Rite in Female Monasteries of the Middle Ages", en HAMBURGER, Jeffrey. F y MARTI, Susan (2008), op. cit, pp. 191-206; MUSCHIOL, Gisela, “Osterliturgie in Frauenklöstern des Mittelalters", en KOLDAU, Linda Maria (coord.), Passion und Ostern in den Lüneburger Klöstern (Ebstorf 2009), Verlag Kloster Ebstorf, 2010, pp. 45-66; MUSCHIOL, Gisela, "Migrating Nuns-Migrating Liturgy? The Context of Reform in Female Convents of the Late Middle Ages", en BERGER, Teresa (ed.), Liturgy in Migration: Cultural Contexts from the Upper Room to Cybserpsace, Minnesota, Liturgical Press, 2012, pp. 83-100; JÄGGI, Carola, "Architecture et disposition liturgique des couvents féminins dans le Rhin supérieur aux XIIIe et XIVe siècles", en BLONDEL, Madeleine, HAMBURGER, Jeffrey F. y LEROY, Catherine (eds.) (2000), op. cit, tomo I, pp. 89-107; JÄGGI, Carola, "Liturgie und Raum in franziskanischen Doppelklöstern. Königs-felden und S. Chiara Neapel im Vergleich", en BOCK, Nicolas, KURMANN, Peter, ROMANO, Serena y SPIESER, Jean-Michel (eds.), Art, Cérémonial et Liturgie au moyen Âge, Actes du 3 er cycle Romand des Lettres (Lausanne, 2000), Roma, Viella, 2002, pp. 223-246; JÄGGI, Carola, Frauenklöster im Spätmittelalter: Die Kirchen der Klarissen und Dominikanerinnen im 13. und 14. Jahrhundert, Petersberg, Michael Imhof Verlag, 2006.

6 CÁTEDRA, Pedro M., Liturgia, poesía y teatro en la Edad Media, Madrid, Gredos, 2005. Sobre las representaciones teatrales en ámbito monástico durante los siglos XVI, XVII y XVIII, así como otro tipo de manifestaciones escritas debidas a las religiosas, a ambos lados del Atlántico vid. ARENAL, Electa y SCHLAU, Stacey, Untold Sisters. Hispanic Nuns in Their Own Works, Alburquerque, University of New Mexico Press, 1989; WEAVER, Elisa, Convent theatre in early modern Italy: Spiritual Fun and Learning for Women, Cambridge, Cambridge University Press, 2002.

7 Para un mayor desarrollo sobre las fuentes y propuestas metodológicas en el estudio de la liturgia de las Dominicas medievales en Castilla, y la aplicación del enfoque metodológico del "giro litúrgico" en el caso concreto de las procesiones asociadas a Completas vid. PÉREZ VIDAL, Mercedes, "La liturgia procesional de Completas en el ámbito de los monasterios femeninos de la Orden de Predicadores en Castilla", en Hispania Sacra [en prensa]. Asimismo, respecto al necesario enfoque de género, vid. también PÉREZ VIDAL, 


\section{¿Liturgistas y dramaturgas?}

En cualquier caso, la nula consideración que han merecido las mujeres a la hora de abordar el estudio de la liturgia hunde sus raíces en los siglos bajomedievales, cuando se trató de limitar su participación, reduciéndolas a meras espectadoras ${ }^{8}$. Así se recoge ya en las actas conciliares a partir del siglo IX, y en diversos tratados de derecho canónico, como Decretum Gratiani, de mediados del XII, que limitó la participación femenina en la Eucaristía, prohibiendo que las religiosas administrasen la comunión, tocasen los vasos sagrados o las vestiduras litúrgicas9. Los reformadores eclesiásticos hicieron especial hincapié en este alejamiento y en la necesidad de una clara separación física, a fin de mantener el decoro en la celebración litúrgica ${ }^{10}$.

Sin embargo, mientras el camaldulense Graciano compilaba su obra en Bolonia, Hildegarda de Bingen desarrollaba en Renania una intensa actividad como predicadora, mística, escritora de un variado corpus literario, y también como compositora de música destinada a la liturgia. Llegó incluso a realizar un drama litúrgico para el Oficio - el Ordo Virtutuum - y muy probablemente también participó en su representación ${ }^{11}$. No solo esto, sino que en su obra visionaria y teológica, Scivias, finalizada poco después de 1151, discutió la viabilidad del sacerdocio femenino, por entonces ya abolido, de forma paralela a la también benedictina Elizabeth de Schönau ${ }^{12}$. Además de estos, existieron otros ejemplos, como los de Catalina Vigri en Bolonia, o Constanza de Castilla, entre otras, que prueban la participación de las monjas tanto en la ordenación de la liturgia, como en la composición de dramas litúrgicos y en la interpolación de elementos ajenos al oficio en romance, a fin de ensalzar las principales festividades del calendario ${ }^{13}$.

Mercedes, "Algunas consideraciones sobre el estudio de la liturgia procesional y paralitugias a través del arte en la Orden de Predicadores en Castilla", Medievalia [en prensa].

8 CLARK, Anne L., "The Priesthood of the Virgin Mary: Gender Trouble in the Twelfth Century", en Journal of Feminist Studies in Religion, CXVIII/1, Spring 2002, pp. 5-24.

9 MACY, Gary, "The Ordination of Women in the Early Middle Ages", Theological Studies, No 61, 2000, pp. 481-507, especialmente p. 495; CLARK, Anne (2002), op. cit., p. 11.

10 ZANOVELLO, Giovanni, “'In oratorio nemo aliquidi agat': Savonarola, lo spazio sacro e la música", en BLOZAM, Jennifer, M., FILOCAMO, Gioia y HOLFORD-STEVENS, Leofranc (eds.), Uno gentile et subtile ingenio: Studies in Renaissance Music in Honour of Bonnie J. Blackburn, Turnhout, Brepols, 2009, pp. 129-136.

11 Aunque no consta documentalmente, Peter Dronke consideró que dicho auto sí habría sido representado, basándose en las acotaciones implícitas del texto y en la coincidencia de las vestimentas de las virtudes descritas en estas y las que aparecen en las visiones de Scivias, que habrían sido tomadas de tal representación. En concreto, la escenificación habría tenido lugar durante la consagración del templo del monasterio de San Rupertsberg en Bingen, en 1152. DRONKE, Peter, "The Text of the Ordo virtutum", en Poetic Individuality in the Middle Ages. New Departures in Poetry, 1000-1150, Oxford, Clarendon Press, 1970, pp. 180-192; DAVIDSON, Audrey Ekdahl (ed.), The "Ordo virtutum" of Hildegard of Bingen: Critical Studies, Kalamazoo, Medieval Institute Publications, 1992; CASTRO CARIDAD, Eva, Dramas Escolares Latinos (siglos XII y XIII), Madrid, Akal, 2001, pp. 83-88. Poco antes, hacia 1150, Hildegarda había reunido setenta y siete composiciones musicales - antífonas, responsorios, himnos, secuencias, etc. - destinadas a su comunidad de Rupertsberg, en una sola obra: Symphonia armonie celestium revelationum. Tales piezas fueron compuestas para ser cantadas en las horas canónicas, en las festividades principales del ciclo litúrgico y en la consagración de alguna capilla. HILDEGARDA DE BINGEN, Sinfonía de la armonía de las revelaciones celestiales, traducción de María Isabel Flisfisch, Madrid, Trotta, 2003.

12 CLARK, Anne L. (2002), op. cit., pp. 12-17.

13 WEAVER, Elisa (2002), op. cit., pp. 49-51. 
En la Península Ibérica, en contraste con la ausencia de teatro vernáculo en Castilla entre mediados del siglo XII - con el aislado ejemplo del Auto de los Reyes Magos toledano - y finales de XV, en los monasterios femeninos fue frecuente la interpolación de composiciones en romance acompañadas de música en la liturgia -"poesía dramática", en palabras de Pedro Cátedra - que acabarían dando lugar a verdaderas representaciones teatrales. Tal finalidad tuvieron, ya en el XV, la Representaçion del Nasçimiento de Nuestro Señor escrita por Gómez Manrique para su hermana, monja en el monasterio de Calabazanos; el anónimo Auto de la huida a Egipto, conservado en un manuscrito procedente del convento de Santa María de las Bretonera; las composiciones insertas en el llamado Cancionero musical de Astudillo, descubierto y estudiado por Cátedra, y las Contemplaçiones que Juan Álvarez Gato. Los tres primeros, y muy probablemente también el último, estuvieron destinados a comunidades de monjas clarisas. Esta proliferación de ejemplos ha llevado a suponer que tal fenómeno de interpolación litúrgica fue propio y prácticamente exclusivo de las clarisas - consideración sobre la que volveré más adelante - vinculándolo, además, a la introducción de la reforma en la segunda mitad del XV. Como es sabido, esta implicó el retorno a la legislación primitiva, incidiéndose con frecuencia en la observancia de la clausura. Precisamente, este encerramiento y aislamiento del mundo al que fueron sometidas las religiosas de los monasterios reformados, ha sido aducido como causa del desarrollo de estas prácticas representativas, que tenían lugar en la más estricta intimidad y que vendrían a reforzar la identidad colectiva de las monjas.

Por otra parte, la introducción de la reforma habría implicado una depuración de la liturgia, con su subsiguiente repercusión en las prácticas paralitúrgicas, musicales y devocionales asociadas a la misma. Como ha señalado Gisela Muschiol, en muchos casos, las monjas enviadas a reformar un determinado monasterio llevaron consigo el rito de su comunidad de origen, dando lugar a una auténtica "migración litúrgica" 14 . En Castilla, sin embargo, a mi juicio, este traspaso de la liturgia no estuvo exclusivamente ligado a la reforma de finales del siglo $\mathrm{XV}$, sino que muy probablemente también ocurrió en fundaciones "filiales" de un determinado monasterio, o bien por influjo de ciertos movimientos que podríamos denominar "ultrarreformistas". Dentro del primer caso podemos considerar el monasterio de Mater Dei, fundado por Constanza de Castilla entre 1449 y 1451, a la sazón priora de Santo Domingo el Real de Madrid. Por concesión expresa del provincial fray Esteban de Sotelo la organización litúrgica de este quedó en manos de la citada Constanza ${ }^{15}$. Tal testimonio pone de manifiesto, además de una más que probable "migración" de la liturgia del monasterio madrileño a la nueva fundación, el papel activo de esta religiosa en la ordenación del rito. Esto resulta palmario también a través de su famoso Libro de Devociones y

14 Se centra en este estudio en el caso de los monasterios de Dominicas en el ámbito germano y en los pertenecientes a las congregaciones de Bursfelde y Windesheim: MUSCHIOL, Gisela (2012), op. cit. pp. $85-100$.

15 "Item que los dias feriados puedan las sorores de mater dei dezir las horas rezadas en la manera que vos ordenaredes e mandaredes": AHN, Libro 7296, Libro de las licencias y gracias que los Sumos Pontifices y ministros generales del orden de Predicadores concedieron a la Serenísma Señora Doña Constanza, nieta del Rey Don Pedro y al Monasterio de Santo Domingo el Real donde fue priora 38 años, siglo XVII, s.f. 
Oficios, compilado por ella misma, y que no solo estuvo destinado a la recitación privada, sino que incluyó algunas partes destinadas a su uso coral, como el Oficio de los Clavos, que se celebraba con licencia pontificia y del Maestro General de la Orden. Asimismo, es posible que otras oraciones y letanías contenidas en este libro hubiesen tenido un uso comunitario y procesiona ${ }^{16}$. En este sentido, Pedro Cátedra ha señalado como con frecuencia la inclusión de estas composiciones en romance - que a partir de los siglos XIV y XV aparecen perfectamente integradas en la liturgia - no en los libros del oficio comunitario, sino en los devocionarios y libros de horas, ha llevado al olvido de su eminente uso ritual ${ }^{17}$.

En lo que respecta a la influencia de los movimientos ultrarreformistas en la liturgia y en las representaciones a ella asociadas, pueden traerse a colación ejemplos como los del predicador savonaroliano fray Niccolò Fabroni, que en uno de sus sermones dedicadas a las Dominicas de San Giorgio di Lucca decía: "sarà occiso Christo, come voi havete rapresentato a giorni passati". Es decir, aludía a una representación paralitúrgica o prototeatral asociada probablemente al ciclo Pascual. Además, la influencia entre los sermones y las liturgias de las religiosas fue recíproca. Si por un lado las paraliturgias y dramatizaciones de la liturgia nutrieron los sermones de los predicadores, por otra, estos incentivaron la práctica de aquéllas ${ }^{18}$. Más llamativo aún es el caso del monasterio de Santo Domingo de Toledo, en el que, como se verá más abajo, el influjo de uno de estos movimientos - liderado por una religiosa - no solo parece haber repercutido en la celebración de tales representaciones, sino que, muy probablemente, condicionó incluso el "escenario" en el que estas se desarrollaron.

\section{Liturgia y teatro en los monasterios de las Dominicas castellanas. A través del arte y la arquitectura}

A pesar de la señalada colaboración interdisciplinar que ha guiado buena parte de la investigación en las últimas décadas, la falta de integración entre el estudio de los textos literarios, por un lado, la liturgia, por otro, y la arquitectura y la cultura visual y objetos devocionales de los monasterios, ha acarreado una serie de problemas. En primer lugar, la ausencia de textos teatrales, o de interpolaciones en la liturgia, ha sido aducida como prueba de la inexistencia de tales representaciones. En segundo, el estudio descontextualizado de tales textos, es decir, sin tener en cuenta el ámbito representativo de los mismos y los objetos que formarían parte del "atrezzo" y, por otro lado, de estos espacios y objetos, sin relación a su función ceremonial y carácter polisémico, ha generado interpretaciones incompletas, cuando no erróneas, de los mismos.

16 LÓPEZ, Juan, Tercera parte de la historia de Santo Domingo y su Orden de Predicadores, libro I, capítulo XXXIIII, Valladolid, 1613; WILKINS, Constance L. (1998), op. cit., p. 342.

17 CÁTEDRA, Pedro M. (2005), op. cit., p. 341.

18 SERVENTI, Silvia, "Prediche di Savonaroliani alle monache di San Giorgio in Lucca", en ZARRI, Gabriela y FESTA, Gianni (eds.), Il Velo, la Penna e la Parola, Florencia, Nerbini, 2009, pp. 217-232, principalmente p. 220. 
Como se ha visto líneas arriba, el que la mayoría de las representaciones o interpolaciones vernáculas en la liturgia conservadas procedan de monasterios de clarisas ha abundado en la consideración del papel preeminente, cuando no exclusivo, de los franciscanos en la génesis de un teatro religioso, fundamentalmente articulado en torno al ciclo navideño y que remontaría su origen al belén de Greccio ${ }^{19}$. Sin embargo, a través no solo de ciertas composiciones poéticas dispersas o insertas de forma marginal en libros de uso comunitario - como el Codex Matritensis de Santo Domingo el Real de Madrid - o privado - Libro de Devociones y Oficios de Constanza de Castilla -, sino también de la liturgia, las evidencias arquitectónicas y ornamentales, y de la "recontextualización" de determinados objetos, podemos formular hipótesis acerca del desarrollo de tales celebraciones también en los monasterios de Dominicas castellanas.

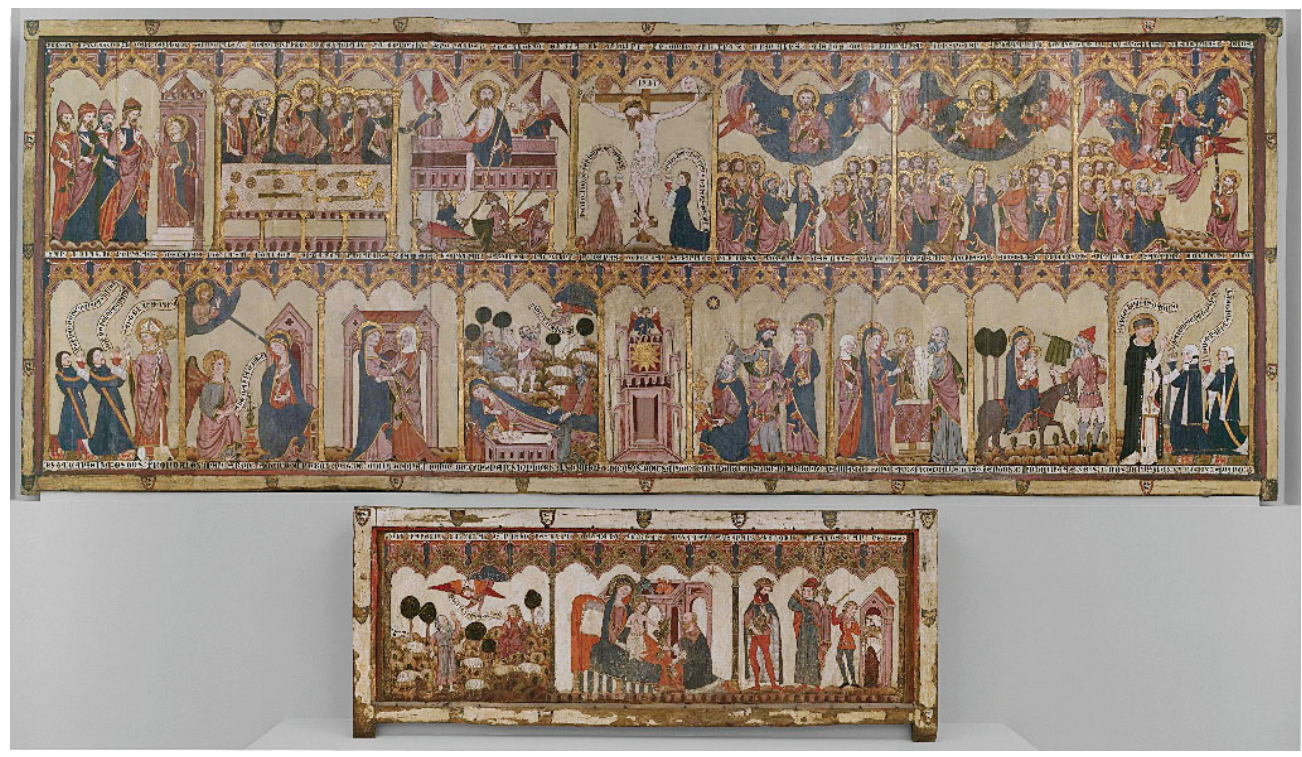

Fig. 1. San Juan Bautista de Quejana. Retablo y Frontal de la Capilla de la Virgen del Cabello. 1396. Actualmente en el Art Institute of Chicago. (Foto: The Art Institute of Chicago. Inv. 1928.817).

En este sentido, la duplicación de las escenas de la Adoración de los Magos y el Anuncio a los Pastores en el frontal de San Juan Bautista de Quejana, ya presentes en el retablo, resulta significativa, dado que normalmente la iconografía de los retablos y frontales concebidos conjuntamente era complementaria y, en consecuencia, no solía repetirse en ellos el mismo episodio (fig. 1). Aunque aquí se ha argüido como motivo de tal reiteración el carácter local de la obra y la escasa disposición de mo-

19 SURTZ, Ronald E., "The Franciscan Connection in the Early Castilian Theater", en Bulletin of the Comendiantes, $\mathrm{n}^{\circ} 35,1983$, pp. 141-152; CÁTEDRA, Pedro M. (2005), op. cit., p. 384. 
delos de taller, a mi juicio esta debió ser intencional ${ }^{20}$. Como ha señalado Schmidt, muchos frontales tempranos realizados en metal eran mostrados únicamente en las festividades principales y no debe descartarse que lo mismo hubiese sucedido con algunas tablas pintadas, como el frontal de Quejana ${ }^{21}$. De ser cierta esta hipótesis, este podría interpretarse como una pieza asociada a la celebración de la Navidad, que sería colocada en la capilla de la Virgen del Cabello con motivo de esta festividad, a la que no es desatinado suponer que se hubiera asociado algún tipo de representación paralitúrgica, de la que, lamentablemente, no hemos conservado testimonio escrito.

Más significativo resulta el monasterio toledano de Santo Domingo el Real, sin parangón dentro de los monasterios medievales de la Orden en Castilla, y que hasta el momento no ha recibido la atención que merece. Las primeras noticias de la celebración de la Navidad en este son de finales del siglo XV y están estrechamente relacionadas con la figura de sor Juana Enríquez de Herrera, monja profesa desde 1472, y que ocupó el cargo de priora en 1480. La documentación evidencia una estrecha vinculación de esta religiosa con los Reyes Católicos, que la habrían empleado en un frustrado intento de reforma del monasterio madrileño de Santo Domingo en $1480^{22}$.

A esta religiosa perteneció una curiosa cuna gótica, probablemente de finales del $\mathrm{XV}$, que constituye un unicum en la Península Ibérica (fig. 2) ${ }^{23}$. En ella aparece escrito en caracteres góticos el siguiente texto: IACET IN PRESEPIO ET IN NUNIBUS TONAT GLORIA IN EXCELSIS DEO ET IN TERA PAX (sic). La primera parte de tal inscripción corresponde a la segunda del responsorio $O$ regem celi, que, de acuerdo con el Ordinario dominico de 1256, aparecía inserto en el tercer nocturno de los maitines de la fiesta de la Circuncisión, al final del cual se cantaba también el Puer Natus est ${ }^{24}$. Según la tradición conventual la celebración de tal festividad estaba acompañada de una serie de paraliturgias, si bien las referencias a esta práctica son tardías ${ }^{25}$. Sin embargo, la segunda parte de la inscripción contiene el Gloria in excelsis, que ponía fin al diálogo pastoril

20 MELERO MONEO, María Luisa, "Retablo y frontal del convento de San Juan de Quejana", en Locus Amoenus, $\mathrm{n}^{\circ}$ 5, 2000-2001, pp. 33-51, especialmente p. 46.

21 SCHMIDT, Victor M., "Ensembles of Painted Altarpieces and Frontals", en KROESEN, Justin y SCHMIDT, Victor M. (eds.), The Altar and its Environment (1150-1400), Turnhout, Brepols, 2009, pp. 203222, especialmente p. 207.

22 Archivo de Santo Domingo el Real de Toledo (en adelante ASDRT), documentos $\mathrm{n}^{\circ} 1112, \mathrm{n}^{\circ} 2192$ y $\mathrm{n}^{\circ}$ 611. ROMERO FERNÁNDEZ-PACHECO, Juan Ramón, Santo Domingo el Real de Madrid. Ordenación económica de un señorio conventual durante la Baja Edad Media (1219-1530), Salamanca, San Esteban, 2008, p. 121; GALÁN VERA, María Jesús, "Toledo oculto: la Navidad en el Convento de Santo Domingo el Real", en CAMPOS Y FERNÁNDEZ SEVILLA, Francisco Javier (coord.), La Navidad: Arte, Religiosidad y Tradiciones Populares, El Escorial, Instituto Escurialense de Investigaciones Históricas y Artísticas, 2009, pp. 123-140.

23 Procedentes fundamentalmente del norte y centro de Europa, hemos conservado objetos semejantes de cronología medieval. Como ejemplos destacados cabe mencionar una cuna de mediados del siglo XIV del Museo Schnütgen de Colonia (A. 776), y otra de la siguiente centuria, procedente del gran Beguinaje de Lovaina, conservada en el Metropolitan de Nueva York (1974.121).

24 Ordinarium iuxta ritum sacri ordinis fratrum praedicatorum, reeditado bajo el generalato de Ludovico Theissling, Roma, Società Tipográfica A. Manuzio, 1921, p. 14. El texto completo dice: "O regem celi, cui talia famulantus obsequia! Stabulo ponitur, qui continet mundum: Iacet in praesepio, et in nubibus tonat".

25 GALÁN VERA, María Jesús, (2009), op. cit., p. 134. 


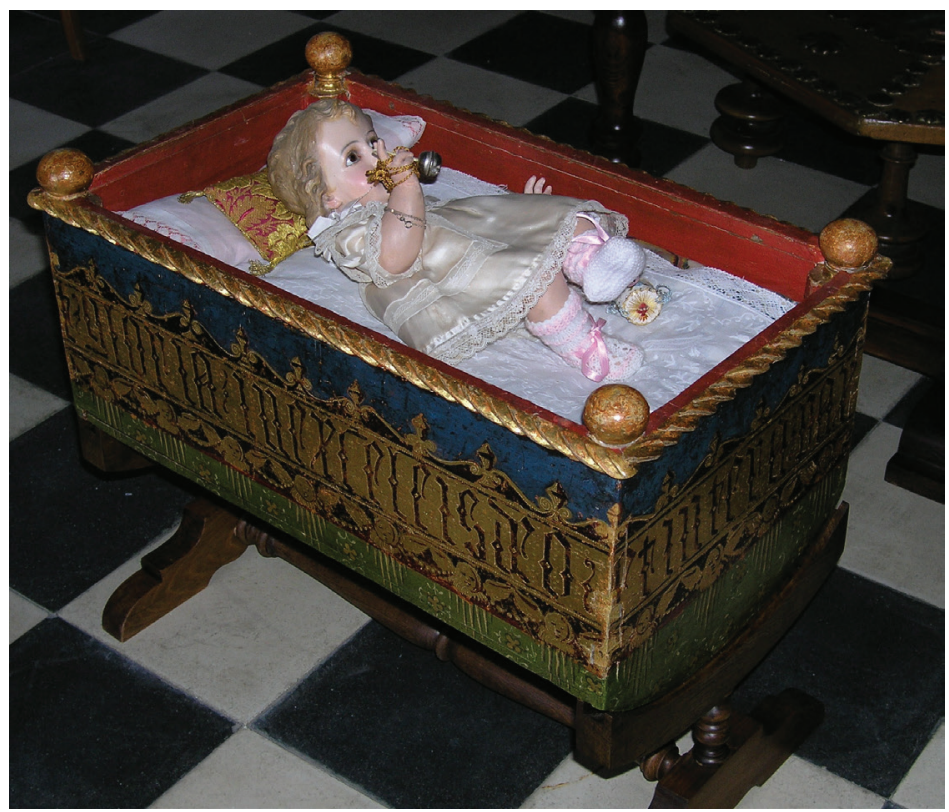

Fig. 2. Santo Domingo el Real de Toledo. Cuna Juana Enríquez de Herrera. (Foto: Archivo de Santo Domingo el Real de Toledo).

inserto en el oficio de laudes del día de Navidad ${ }^{26}$. A todo esto debemos añadir el Romance del Nascimiento de nuestro Salvador compuesto en 1485 por fray Ambrosio de Montesino, a petición de la misma sor Juana de Herrera, y que fue incluido en su cancionero publicado en Toledo en $1508^{27}$. Este poema estuvo probablemente acompañado de música, como sucedió con otros debidos al franciscano, empleándose la técnica del contrafactum, es decir, reutilizando melodías preexistentes, normalmente populares, no religiosas. Como se ha visto líneas arriba, la inserción de composiciones líricas en el oficio fue algo habitual en la Baja Edad Media, como prueba la tradición de los gozos dedicados a la Virgen o los santos, o las laude italianas ${ }^{28}$.

A mi juicio, todos estos elementos nos están hablando claramente de la celebración de la Navidad con cierto grado de teatralización en el monasterio toledano. Esta

26 Ordinarium iuxta ritum sacri ordinis (1921), op. cit., p. 12

27 Este cancionero constituyó una versión ampliada de una colección de poemas, publicada por primera vez en 1485 con el título Coplas sobre devoçiones y misterios de nuestra sancta fe catholica, cuyo único ejemplar conservado se encuentra en la British Library. MONTESINO, Ambrosio, Cancionero de diversas obras de nuevo trobadas: todas compuestas hechas y corregidas por el padre Fray Ambrosio Montesino de la orden de los menores, Valencia, Vicente García Editores, 2002 (1508). Fray Ambrosio también escribió poemas para las abadesas de San Clemente, Santo Domingo el Antiguo, Santa Isabel, los Reyes Católicos y el Cardenal Cisneros, entre otros.

28 Estas últimas se convirtieron en la expresión musical por excelencia del movimiento savonaroliano, siendo cultivadas también en los monasterios femeninos vinculados al mismo. MACEY, Patrick, "La música nei conventi savonaroliani nel Cinquecento", en ZARRI, Gabriella y FESTA, Gianni (eds.) (2009), op. cit., pp. 177-184, especialmente pp. 180-182. 


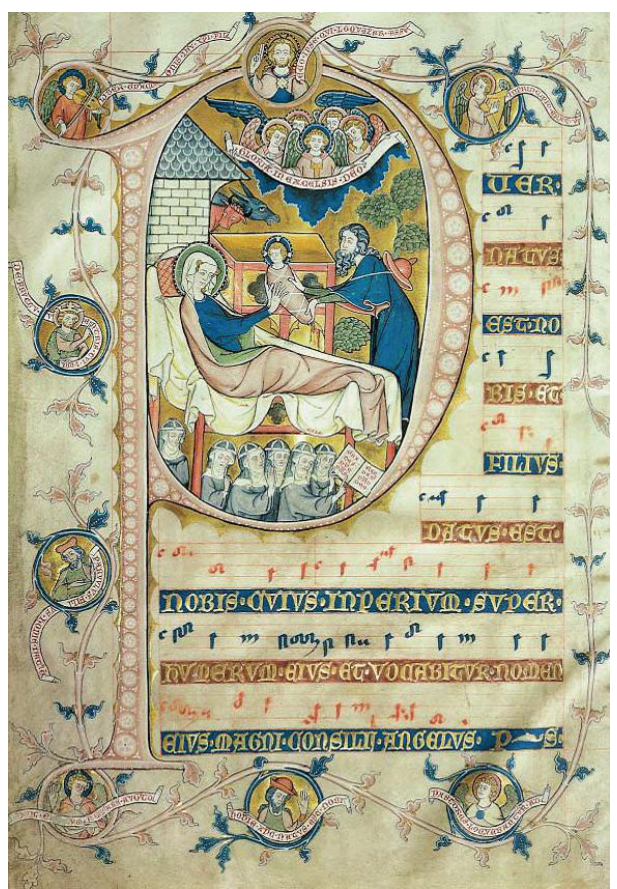

Fig. 3. Gradual de Gisela von Kerssenbrock ca. 1300, f. 25. Procedente del monasterio de Marienbrunn (Foto: Museo diocesano de Osnabrück).

habría tenido posiblemente como modelo las representaciones de la sede primada, en las que no existió un Officium Pastorum en los maitines de Navidad, sino que al final de este oficio se insertó el canto de la Sibila, que proseguía sin solución de continuidad en el diálogo de los pastores de laudes, concluyendo con el canto del Gloria y con la inserción de coplas en romance, villancicos y danzas al final del mismo, al menos desde finales del siglo $\mathrm{XV}^{29}$. Según todo esto, y teniendo en cuenta que en los monasterios femeninos la contaminación de la tradición litúrgica local fue frecuente, considero probable que el romance de Montesino, en el que se refiere el anuncio del Nacimiento de Cristo a los pastores por el Ángel y la posterior adoración de aquellos, fuese interpolado en el curso del oficio de laudes del día de Navidad, y dramatizado, quizás en sustitución del diálogo pastoril, finalizando todo ello con el Gloria recogido en la cuna. Además, como se ha visto, la inserción en la liturgia comunitaria de composiciones destinadas a una religiosa no fue infrecuente, tal y como sucedió en el caso de Constanza de Castilla, o con laude de Santa Catalina Vigri, la cual tuvo un papel activo en la ordenación litúrgica, destacando también la inserción de composi-

29 SURTZ, Ronald E., The Birth of a Theater. Dramatic convention in the Spanish theater from Juan del Encima to Lope de Vega, Princeton/Madrid, University of Princeton y Castalia, 1979, p. 17; DONOVAN, Richard B., The Liturgical Drama in Medieval Spain, Toronto, Pontifical Institute of Mediaeval Studies, 1958, pp. 34-37. 


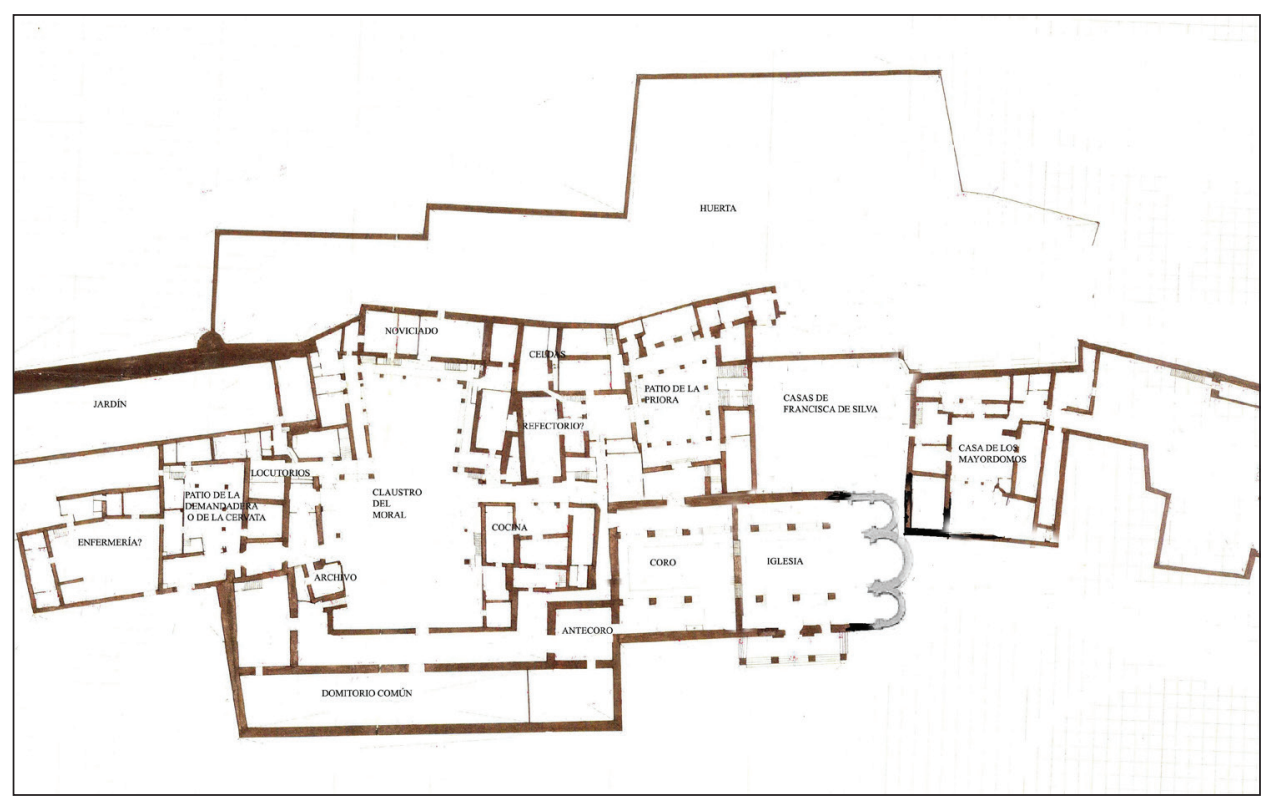

Fig. 4. Santo Domingo el Real de Toledo. Reconstrucción del estado del monasterio a inicios del siglo XVI con la nueva panda meridional del claustro del Moral (1507-1508). (M. Pérez Vidal sobre el plano del instituto Geográfico y Estadístico, 1881).

ciones sobre el Nacimiento ${ }^{30}$. Es probable que en su representación se emplease una cuna similar a la toledana, acompañada de una imagen de Jesús Infante, que también debió existir en Toledo, y que ha sido sustituida por otra de cronología posterior.

Una miniatura del gradual de Gisela von Kerssenbrock, realizado por esta religiosa y conservado en el museo diocesano de Osnabrück, parece constituir una representación visual de tales representaciones, evidenciando además el carácter contemplativo de las mismas (fig. 3). Se encuentra inserta en la inicial del introito de la tercera misa del día de Navidad - Puer Natus est - y en ella además de representarse la escena del Nacimiento en el establo, con el coro angélico en la parte superior que entona el Gloria, vemos un grupo de religiosas situadas bajo el pesebre y frente a un cantoral abierto $^{31}$. Cabe destacar aquí dos cosas: su origen cisterciense y su temprana cronología, pues fue realizado en torno al 1300 para el monasterio de Marienbrunn.

Pero volviendo al monasterio toledano, una vez desmenuzados, en la medida de lo posible, los entresijos de la celebración navideña en el mismo, resta conocer dónde tenía lugar tal representación, algo que no se ha planteado hasta la fecha, ni en este ni en otros casos. Entre el coro de las religiosas y la panda meridional del claustro del

30 WOOD, Jeryldene M., "Breaking the Silence: The Poor Clares and the Visual Arts in Fifteenth-Century Italy”, en Renaissance Quarterly, XLVIII-2, 1995, pp. 262-286, especialmente pp. 272-273

31 Gradual de Gisela von Kerssenbrock, ca. 1300, f. 25. Domschatzkammer und Diözesanmuseum Osnabrück. OLIVER, Judith H., Singing with Angels. Liturgy, music and art in the Gradual of Gisela von Kerssenbrock, Turnhout, Brepols, 2007. 


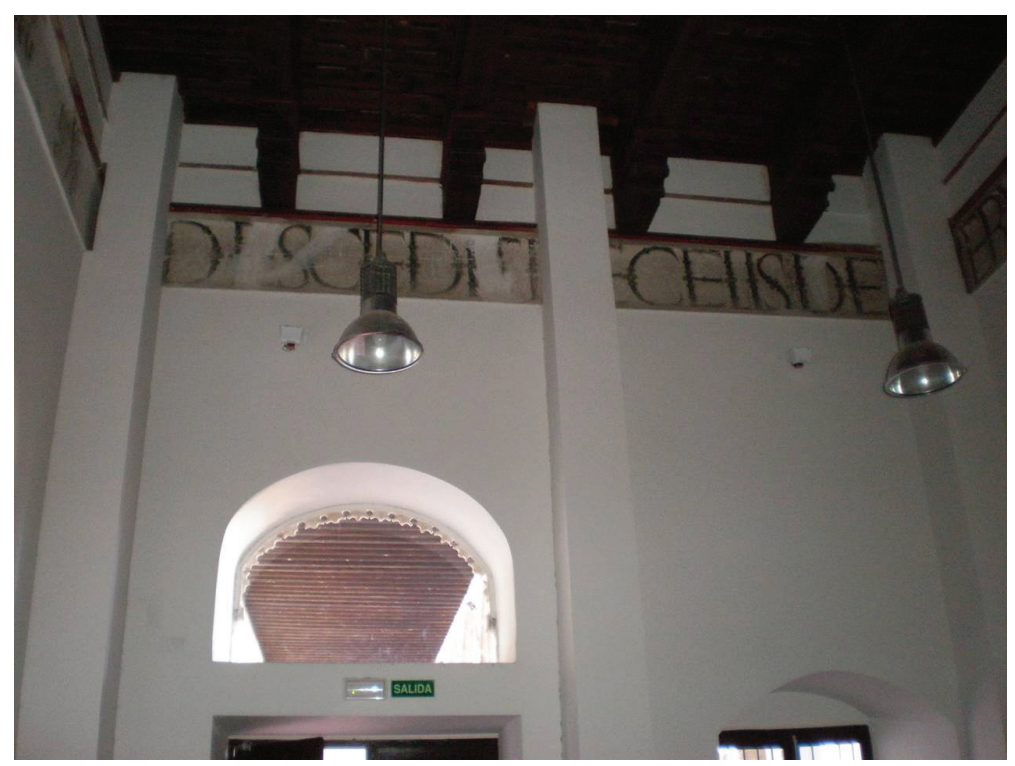

Fig. 5. Santo Domingo el Real de Toledo. Dependencia entre coro y claustro del Moral, conocida como "sacristía vieja". Inscripción de los maitines de Navidad (Foto: M. Pérez Vidal).

Moral se encuentra un espacio cuadrado, de notables dimensiones y de dudosa funcionalidad, denominado tradicionalmente "sacristía vieja" (figs. 4 y 5). Según Martínez Caviró, este habría sido construido de forma contemporánea a la citada panda meridional - iniciada en 1507, según la documentación monástica e interrumpida, quedando inconclusa, en 1508. Así lo prueban los vanos que dan acceso al claustro, las jácenas que aún conserva y la inscripción del friso que recorre sus cuatro muros, bajo el alfarje.

El texto de este es fácilmente legible en los muros oeste, norte y parte del este: DESCENDIT DE CAELIS DEUS VERUS A PATRE GENITUS: INTROIVIT IN UTERUM VIRGINIS. Según el ordinario dominico de 1256, corresponde al tercer responsorio del primer nocturno de los maitines de Navidad, tras el Gloria y versículo Hodie Illuxit dies redemptoris nostrae, y seguido del Tamquam sponsus Dominus procedens de thalamo suo, y de nuevo el Gloria $^{32}$. Por otra parte, la elección de tal texto debió estar también motivada por su clara alusión a la Encarnación, que en los siglos bajomedievales era considerada el primer derramamiento de sangre redentora, prefigurando la Pasión ${ }^{33}$. En la

32 Ordinarium iuxta ritum (1921), op. cit, p. 11. El texto completo del responsorio dice: "Descendit de caelis Deus verus a Patre genitus, introivit in uterum Virginis, nobis tu apparet visibilis, indutus carne humana protopartente edita; et exivit per clausam portam Deus et homo lux et vita, conditor mundi".

33 Gertrude de Hefta tuvo una visión de una Virgen con el niño Dios Encarnado en su seno -probablemente una representación de María Grávida - tras el canto de este mismo responsorio, HAMBURGER, Jeffrey F. (1998), op. cit., p. 118. Cabe recordar también que el citado libro de Devociones y Oficios de Constanza de Castilla fue compilado "en memoria de tu Encarnación y tu Pasión". BNE, Ms. 7495, f. 82 v. WILKINS, Constance L., "En memoria de tu encarnación e pasión”. The Representation of Mary and Christ in the Prayer 
nave del Evangelio del coro del monasterio toledano existió un altar dedicado a la Encarnación, al menos desde 1561, momento en que Pío IV concedió a las monjas la celebración de tal festividad como jubileo ${ }^{34}$. A mi juicio, esto vendría a explicar también la inclusión de parte del citado responsorio $O$ regem celi en la cuna toledana, vinculando el momento de la adoración del pesebre por los ángeles, al anunció de su futura Pasión. Tal asociación se convirtió en una constante en la literatura devocional en torno al Nacimiento, con la aparición de los Arma Christi, que los ángeles mostraban al Niño, integrándose también en las representaciones teatrales, como prueban el Libro del Conhorte, de la franciscana Juana de la Cruz, o la Representaçión de Gómez Manrique ${ }^{35}$. En el caso toledano, Montesino aludía a los futuros tormentos y, además, los instrumentos de la Pasión aparecen representados también en los vanos de esta panda claustral, y en un cáliz datado también entre finales del siglo XV e inicios del XVI ${ }^{36}$.

Así pues, descartada su función como sacristía, no solo por todos los elementos referidos, sino también por su ubicación entre el coro y el claustro, sin acceso por parte de los sacerdotes ${ }^{37}$, resulta claro que la mencionada dependencia debió tener otras funciones. Entre ellas bien pudo haber estado la celebración de la liturgia de maitines y laudes del día de Navidad, con la inserción en este último oficio del poema de Montesino, y quizás también de otras composiciones.

Por otra parte, junto al innegable influjo franciscano- a través de Montesino-y de la sede primada en las representaciones del monasterio toledano, no debe descartarse tampoco el debido a uno de los movimientos"ultrarreformistas" anteriormente aludidos, el organizado en torno a la Beata de Piedrahita. Como he analizado en otro lugar, varias características de esta panda concuerdan perfectamente con la espiritualidad y religiosidad de la Beata, que se caracterizó por la devoción, meditación y penitencia en torno a la Pasión, el gusto por la profusión de procesiones, y por la importancia de la música, empleando un canto más solemne y prolongado que el habitual en la $\operatorname{Orden}^{38}$. A esto cabe sumar que las fechas de inicio y cese de las obras -1507 y 1508 -

book by Sor Constanza de Castilla", en La Corónica. A Journal of Medieval Hispanic Languages, Literatures and Cultures, vol. XXXI, n ${ }^{\circ}$ 2, 2003, pp. 217-235, especialmente p. 220.

34 ASDRT, doc. 1232, dada el 30 de agosto de 1561.

35 GARCÍA ANDRÉS, Inocente, "El "Conhorte". Sermones de una mujer, la santa Juana (1481-1534), Madrid, FUE, 1999, tomo I, p. 263; SURTZ, Ronald E., Teatro Castellano de la Edad Media, Madrid, Taurus, 1992, pp. 82-86.

36 "Lloró con bajos clamores/Por ensayarse en el heno/A otros plantos mayores/Con los cuales dio su alama/En la cruz por mis errores": MONTESINO, Ambrosio, (2002), op. cit.; MARTÍNEZ-BURGOS GARCÍA, Palma (coord.), Dominicas. VIII Centenario, Toledo, 2007, p. 252.

37 GILCHRIST, Roberta, Gender and Material Culture. The Archaeology of Religious Women, London y New York, Routledge, 1994, p. 111.

38 PÉREZ VIDAL, Mercedes, "Observancia y rigorismo. Consecuencias de la reforma de la Orden de Predicadores y de algunos movimientos rigoristas en la liturgia y arquitectura de los monasterios de dominicas de la "Provincia de España", en FERNÁNDEZ RODRÍGUEZ, Natalia y FERNÁNDEZ FERREIRO, María (eds.), Literatura medieval y renacentista en España: líneas y pautas. Actas del III Congreso internacional de la SEMYR (Oviedo, 2010), Salamanca, SEMYR, 2012, pp. 799-810. La Beata consideraba que cuando el alma se hallaba libre de pecado por medio de la confesión y la penitencia, la música podía ser muy beneficiosa, pues a través de ella se alcanzaba la armonía con Cristo. GILES, Mary E., The Book of Prayer of Sor María of Santo Domingo. A Study and Translation, Albany, State University of New York, 1990, pp. 174-176. 
coinciden respectivamente con el envío de la Beata a Toledo por orden del provincial Diego Magdaleno y la prohibición de su actividad predicadora ${ }^{39}$. En última instancia, el nexo entre todas estas influencias-Montesino, la Beata y la sede primada toledanahabría sido el cardenal Cisneros.

Ahora bien, ¿existieron espacios similares en otros monasterios dominicos? Como se ha visto líneas arriba, el monasterio madrileño de Santo Domingo conserva aún un fascinante códice misceláneo - el Codex matritensis - compuesto por varias partes realizadas entre finales del XIV e inicios del $\mathrm{XV}^{40}$. Además de contener los oficios de las dos fiestas de santo Tomás, escritos en romance en su totalidad, versificados y con una clara finalidad representativa, incluyó también los legenda, también en romance de este santo, santo Domingo y san Pedro Mártir. Estos constituyeron el texto de las nueve lecciones en prosa que se leían o salmodiaban en el oficio de maitines de sus respectivas fiestas, práctica que fue habitual en la liturgia monástica, como prueban otros ejemplos, como los de Unterlinden, Roma, y los monasterios boloñeses de Val di Piedra y Santa Inés ${ }^{41}$.

¿Dónde tenían lugar tales celebraciones en el monasterio madrileño? ¿Existió, de forma similar a Toledo, un espacio determinado para ellas? Según las Constituciones de las monjas dominicas de 1259, en los monasterios debía existir un lugar en el que las monjas se reunirían antes de entrar en el coro para disponer lo que había de decirse en el Oficio Divino: Aliquis autem locus statuatur. in quo ad preuidendum officium diuinum sorores conueniant: presente priorissa uel alia cui commiserit tempore oportuno ${ }^{42}$. En el monasterio madrileño existieron sendos antecoros, dispuestos a ambos lados del coro, aunque lo más probable es que en el momento en que fue compilado el códice, únicamente existiese el del lado de la Epístola, siendo el otro producto de una ampliación posterior, probablemente ya entrado el XVI (fig. 6) ${ }^{43}$.

39 BELTRÁN DE HEREDIA, Vicente, Historia de la Reforma de la Provincia de España (1450-1550), Roma, Instituto Histórico Dominicano, 1939, pp. 251-253.

40 CÁTEDRA, Pedro. M. (2005), op. cit., p. 384; ALONSO GETINO, Luis G., "Los primeros versos castellanos acerca de Santo Tomás de Aquino", en La Ciencia Tomista, no 68, 1921, pp. 145-159; Idem, Leyenda de Santo Tomás de Aquino (s. XIV), Madrid, 1924, pp. 7-12.

41 HAMBURGER, Jeffrey F., "Le Liber miraculorum d'Unterlinden: une icône dans l'écrin de son couvent", en BLONDEL, Madeleine, HAMBURGUER, Jeffrey F. y LEROY, Catherine (eds.) (2000), op. cit., vol. I, pp. 190-225; KOUDELKA, Vladimir J., "Le monasterium tempuli et la fondation dominicaine de San Sisto", en Archivum Fratrum Praedicatorum, no 31, 1961, pp. 5-8; RONCROFFI, Stefania, Psallite Sapienter. Codici Musicali delle Domenicane Bolognesi, Florencia, Leo. S. Olschki Editore, 2009.

42 Constitutiones Sororum Ordinis Fratrum Praedicatorum (1259), "De officio ecclesie", p. 2. Constitutiones et Acta Ordinis Fratrum Praedicatorum, Berlín, Digitale Bibliothek Spezial, 2002, p. 111 (cf. Const O-P1259, p. 339)

43 En 1538 las monjas traspasaron la capilla del lado del Evangelio a Alonso de Castilla, iniciándose las obras al año siguiente. En este momento debió levantarse también la nave situada frente a ella y el espacio polifuncional situado a los pies de la misma, en el que se dispondría también una tribuna o coro alto y que, posteriormente, funcionó como capítulo: ESTELLA MARCOS, Margarita, "Los artistas de las obras realizadas en Santo Domingo el Real y otros monumentos madrileños de la primera mitad del siglo XVI", en Anales del Instituto de Estudios Madrileños, vol. XVII, 1980, pp. 41-65, especialmente pp. 45-47; ANDRÉS MARTÍNEZ, Gregorio de, "La capilla funeraria de Don Alonso de Castilla, Obispo de Calahorra en Santo Domingo el Real de Madrid", en Anales del Instituto de Estudios Madrileños, no 35, 1995, pp. 293-304 


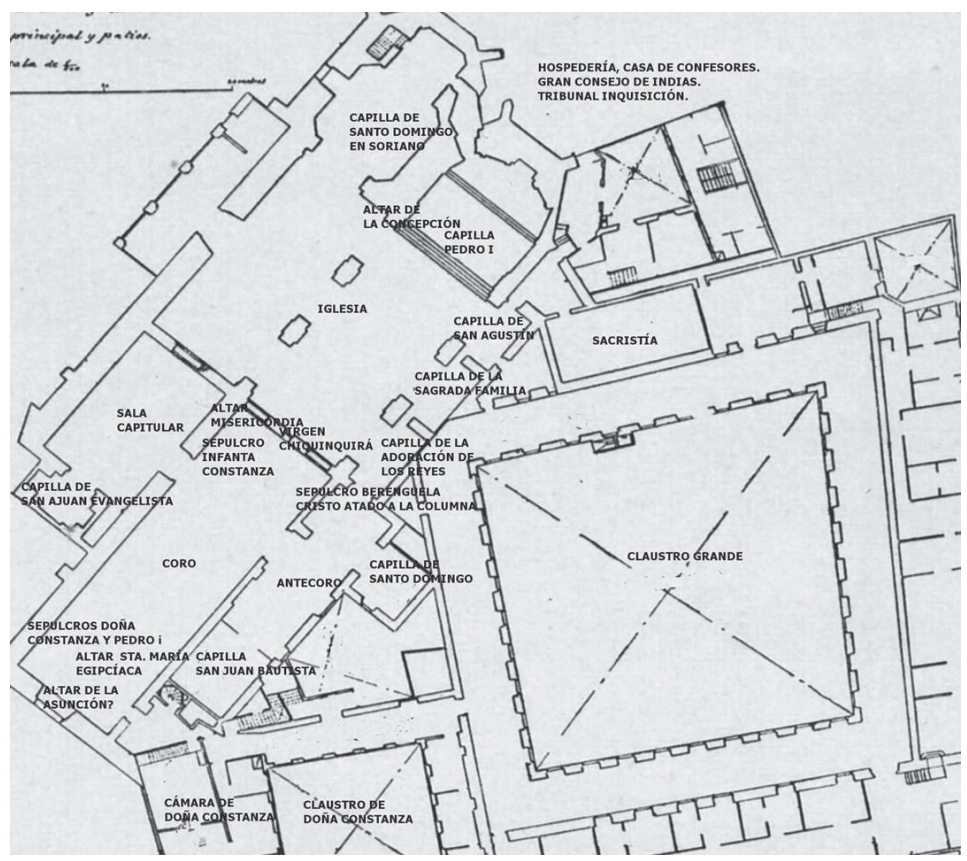

Fig. 6. Santo Domingo el Real de Madrid. Detalle de altares y capillas. (M. Pérez Vidal sobre el plano del Museo Municipal de Madrid. IN. 2695).

En el primero, cuyo origen debió estar en el primer oratorio o iglesia de las monjas, se documentan una serie de altares y capillas que, junto a un zócalo corrido de azulejos con asientos para las religiosas, evidencian que, más allá de una mera función preparatoria del oficio, este espacio habría servido de escenario a varias celebraciones litúrgicas. En su costado meridional se abría la capilla de Santo Domingo, que ocupaba un papel muy destacado en la liturgia procesional del monasterio madrileño, mientras que en la zona meridional del antecoro se encontraba otra capilla destinada a san Juan Bautista. Además, también se documenta aquí una imagen de san Pedro Mártir y es probable, que al menos en un primer momento, existiese otro altar dedicado a santo Tomás, que junto con el de san Pedro y santo Domingo contó con una serie de indulgencias ${ }^{44}$. Según todo esto, no resulta descabellado suponer que las festividades de estos tres santos se hubiesen desarrollado en este antecoro, empleando los altares y las capillas abiertas en el mismo y recitándose en ella los textos contenidos en el códice. Además, al menos en la fiesta de santo Domingo, los frailes contaban con autorización pontificia para penetrar en la clausura.

Fuera de estos ejemplos, no tenemos testimonios de la existencia de espacios semejantes en los monasterios de dominicas castellanos durante el período bajomedieval, ni tampoco en los albores de la Edad Moderna, momento en el que los testimonios,

44 Archivo del Monasterio de Santo Domingo el Real de Madrid, carpeta de bulas, $\mathrm{n}^{\mathrm{o}} 1 \mathrm{y} \mathrm{n}^{\mathrm{o}} 26$. 


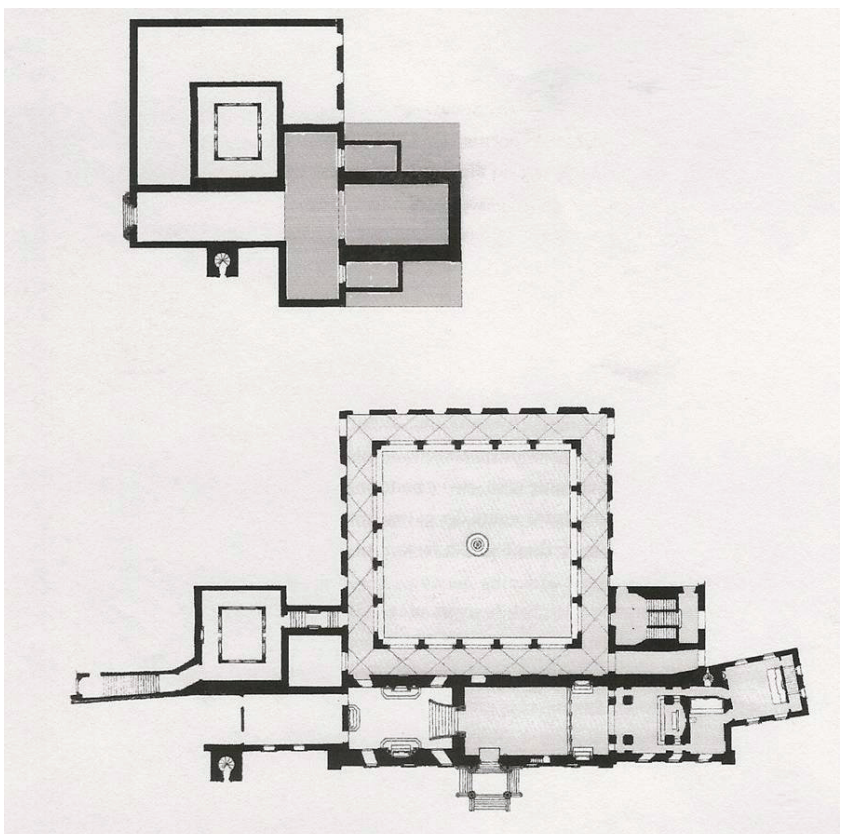

Fig. 7. Madre de Deus de Lisboa. Propuesta planta original, arriba, y estado tras la campaña de Diogo de Torralva, debajo. [Tomado de SIMÕES, João Miguel., "O modelo arquitectónico das duas primeiras casas coletinas portuguesas: os mosteiros de Jesus de Setúbal e da Madre de Deus de Xabregas", en CURVELO, A. (ed)., Casa Perfeitíssima: 500 anos da Fundação do Mosteiro da Madre de Deus, 1509-2009, Lisboa, 2009, p. 70.]

no ya de interpolaciones en la liturgia, sino de auténticas representaciones teatrales en el interior de las clausuras se multiplican. Un ejemplo lo constituye la representación del Auto de la Sibila Casandra, de Gil Vicente, en el monasterio lisboeta de Madre de Deus, en los maitines de la Navidad de 1513, que finalizaba también con la adoración de un pesebre o aparato do nacimiento ${ }^{45}$. Esta debió tener lugar en un espacio convenientemente oculto a la vista de los fieles, dado que, junto a la fundadora - la "rainha velha" Leonor - participaron las propias religiosas, que bien pudieron haber sido los aposentos de la reina, o el coro de las religiosas, situado en un brazo del transepto (fig. 7) ${ }^{46}$. Posteriormente, ya en plena Edad Moderna, una sala aneja al antecoro, se dedicó a la conservación del magnífico belén del monasterio lisboeta, pero no consta su existencia anterior, ni tanto menos su utilización en representaciones asociadas a la Navidad ${ }^{47}$.

45 VICENTE, Gil, Cassandra, Lisboa, Quimera, 2005, pp. 4-5.

46 PAIS, Alexandre y CURVELO, Alexandra, "Memórias da Fogueira. O primitivo mosteiro da Madre de Deus", en CURVELO, Alexandra (ed.), Casa Perfeitíssima: 500 anos da Fundação do Mosteiro da Madre de Deus, 1509-2009, Lisboa, Ministério da Cultura/Instituto dos Museus e Conservação /Museu Nacional do Azulejo, 2009, p. 81.

47 NOBRE PAÍS, Alexandre, PORTUGAL ROMÃO, Laura y JOÃO VILHENA, Maria, O Presépio da Madre de Deus, Lisboa, Museu Nacional do Azulejo, 2003. 


\section{Algunas reflexiones finales}

A lo largo de los siglos bajomedievales las monjas tomaron parte activa en la ordenación de la liturgia, tanto a nivel individual como de forma colectiva, así como en las paraliturgias o representaciones asociadas a la misma. Con respecto al origen de estas últimas, si bien la introducción de la observancia implicó cambios en la liturgia y vida comunitaria que pudieron haberlas incentivado, ejemplos más tempranos como los de las cistercienses de Marienbrunn, o las dominicas de Roma, Bolonia o Madrid, parecen probar que tales interpolaciones en la liturgia fueron en muchos casos anteriores a la reforma. Asimismo, frente a la tradicional suposición de un papel preeminente, cuando no exclusivo de Franciscanos y Clarisas en la génesis del teatro religioso navideño, tanto los ejemplos hasta aquí expuestos como los documentados en algunos conventos masculinos, evidencian que también los Predicadores tuvieron un papel destacado en estas cuestiones ${ }^{48}$.

La "recontextualización" y el estudio conjunto de algunos artefactos que formaron parte de la cultura visual, devocional y representativa de las religiosas, ciertos textos litúrgicos y paralitúrgicos y del espacio arquitectónico, permiten avanzar nuevas hipótesis sobre tales celebraciones, desde una visión integradora. De igual modo que no podemos entender el espacio con independencia de su función, difícilmente podemos entender los textos poéticos, dramáticos o litúrgicos sin relación a su contexto representativo. En el caso de los monasterios además, ya se tratase de espacios preexistentes reutilizados, o de otros de nueva construcción, las monjas parecen haber dispuesto de espacios que, entre otras funciones, tuvieron la de acoger el desarrollo de tales representaciones.

\footnotetext{
48 Galvanno Fiamma relataba en su crónica la representación de la Adoración de los Reyes Magos que desde 1336 tenía lugar en Milán, partiendo de Santa Maria delle Grazie y concluyendo, tras un recorrido urbano, en Sant'Eustorgio, donde se escenificaba la adoración GILLET, Louis, Histoire artistique des ordres mendiants. Études sur l'Art en Europe du XIIIe-XVIIIe siècles, Paris, Librairie Renouard, H. Laurens, 1912, p. 107. En Florencia se celebraba, al menos desde 1390, la Festa de' Magi e della stella, que partía del baptisterio de San Giovanni y discurría por toda la ciudad hasta la chiesa de'fratj di santo Marcho, donde se representaba la Adoración. HATFIELD, Rab, "The Compagnia de' Magi", en Journal of the Warburg and Courtauld Institutes, vol. XXXIII, 1970, pp. 107-161.
} 